\title{
Joint ergodicity for group actions
}

\author{
VITALY BERGELSON† AND JOSEPH ROSENBLATT $\ddagger$ \\ The Ohio State University, Columbus, Ohio 43210, USA
}

(Received 14 July 1986 and revised 23 April 1987)

\begin{abstract}
Let $T_{1}, \ldots, T_{n}$ be continuous representations of a $\sigma$-compact separable locally compact amenable group $G$ as measure-preserving transformations of a non-atomic separable probability space $(X, \beta, m)$. Let $\left(K_{n}\right)$ be a right Følner sequence of compact sets in $G$. If $T_{1}, \ldots, T_{n}$ are pairwise commuting in the sense that $T_{i}(g) T_{j}(h)=T_{j}(h) T_{i}(g)$ for $i \neq j$ and $g, h \in G$, then necessary and sufficient conditions can be given, in terms of the ergodicity of certain tensor products, for the following to hold: for all $F_{1}, \ldots, F_{n} \in L_{\infty}$, the sequence $A_{N}(x)$ where

$$
\begin{aligned}
& A_{N}(x)= \\
& \quad\left(1 /\left|K_{N}\right|\right) \int_{K_{N}} F_{1}\left(T_{1}(g)^{-1} x\right) F_{2}\left(T_{1}(g)^{-1} T_{2}(g)^{-1} x\right) \cdots F_{n}\left(T_{1}(g)^{-1} \cdots T_{n}(g)^{-1} x\right) d g
\end{aligned}
$$
\end{abstract}

converges in $L_{2}(X)$ to $\prod_{i=1}^{n} \int F_{i} \mathrm{dm}$. The necessary and sufficient conditions are that each of the following representations are ergodic: $T_{n}, T_{n-1} \otimes T_{n-1} T_{n}, \ldots, T_{2} \otimes$ $T_{2} T_{3} \otimes \cdots \otimes T_{2} \cdots T_{n}, T_{1} \otimes T_{1} T_{2} \otimes \cdots \otimes T_{1} \cdots T_{n}$.

In order to prove this theorem, specific properties of the decomposition of $L_{2}(X)$ into its weakly mixing and compact subspaces with respect to a representation $T_{i}$ are needed. These properties are also used to prove some generalizations of wellknown facts from ergodic theory in the case where $G$ is the integer group $Z$.

\section{Introduction}

In [7], Furstenberg proved a fundamental theorem on multiple recurrence of measure preserving systems. He showed that for any measure preserving system $(X, \beta, m, T)$, for any $k \geq 1, A \in \beta$, and $m(A)>0$; there exists $n \geq 1$ such that $m\left(\bigcap_{i=1}^{k} T^{-i n} A\right)>0$. In the special case where $T$ is weakly mixing, more was proved: $T$ is weakly mixing of all orders. That is, if $T$ is weakly mixing, then for all $A_{0}, \ldots, A_{k} \in \beta$,

$$
\lim _{N \rightarrow \infty}(1 / N) \sum_{n=1}^{N}\left[m\left(A_{0} \cap T^{-n} A_{1} \cap \cdots \cap T^{-k n} A_{k}\right)-\prod_{i=0}^{n} m\left(A_{i}\right)\right]^{2}=0 .
$$

Indeed, Furstenberg uses (1) as part of the proof in [7]. See also Furstenberg, Katznelson and Ornstein [10] for a discussion of this connection.

As in [10], (1) can be shown by proving for weakly mixing $T$, that all $F_{1}, \ldots, F_{k} \in$ $L_{\infty}(X)$,

$$
\lim _{N \rightarrow \infty}\left\|(1 / N) \sum_{n=1}^{N}\left(\prod_{l=1}^{k} T^{l n} F_{l}\right)-\prod_{l=1}^{k} \int F_{l} d m\right\|_{2}=0 .
$$


This was generalized by Furstenberg and Katznelson in [9], see also Furstenberg [8], in the process of proving an ergodic Szemeredi theorem for commuting transformations. They show that if $S_{1}, \ldots, S_{n}$ are commuting transformations with $S_{i} S_{j}^{-1}$ weakly mixing for all $i \neq j$, then for all $F_{1}, \ldots, F_{k} \in L_{\infty}(X)$,

$$
\lim _{N \rightarrow \infty}\left\|(1 / N) \sum_{n=1}^{N}\left(\prod_{l=1}^{k} S_{l}^{n} F_{l}\right)-\prod_{l=1}^{k} \int F_{l} d m\right\|_{2}=0 .
$$

The hypotheses that are necessary and sufficient for (3) are given in Berend and Bergelson [1] where it is shown that (3) holds for commuting $S_{i}$ if and only if $S_{1} \otimes \cdots \otimes S_{n}$ is ergodic and $S_{i} S_{j}^{-1}$ is ergodic for each $i \neq j$. This latter jointly ergodic criteria is much weaker than assuming $S_{1}, \ldots, S_{n}$ are weakly mixing (for instance, let $S_{i}$ be irrational rotations by rationally independent angles). See also Berend and Bergelson [2] where necessary and sufficient criteria for (3) are given when $T_{1}, \ldots, T_{n}$ do not commute.

In $\S 2,(3)$ is generalized by replacing each $S_{i}$ by commuting actions $S_{i}(g)$ of a general $\sigma$-compact separable amenable locally compact group. First, in $\S 1$, some facts about representations of a $\sigma$-compact locally compact group $G$ as measure preserving transformations are derived which are generalizations of similar theorems for abelian groups $G$. These are then used in $\S 2$ to give the joint ergodicity conditions on commuting actions $S_{i}$ of $G$ which are necessary and sufficient for the generalization of (3).

\section{Groups of measure-preserving transformations}

Assume $G$ is a $\sigma$-compact locally compact Hausdorff group (called a group in the sequel). Let $T: G \rightarrow M(X)$ be a homomorphism of $G$ into the invertible measurepreserving transformations $M(X)$ of a probability space $(X, \beta, m)$. For $F: X \rightarrow C$, $g \in G,(T(g) F)(x)=F\left(T(g)^{-1} x\right)$ for all $x \in X$. A representation $T$ of $G$ in $M(X)$ will be any such homomorphism for which the mapping $g \rightarrow \int T(g) F_{1} F_{2} d m$ is continuous for all $F_{1}, F_{2} \in L_{2}(X)$. Generally, $L_{2}(X)$ is a direct sum of two orthogonal closed subspaces, denoted here by $L_{2}(X)_{w}$ and $L_{2}(X)_{c}$. The compact summand $L_{2}(X)_{c}$ consists of all $F \in L_{2}(X)$ such that $\{T(g) F: g \in G\}$ is totally-bounded in $L_{2}(X)$. Let $M$ denote the unique $G$-invariant mean on WAP $(G)$, the weakly almost periodic functions on $G$. Then the weakly mixing part $L_{2}(X)_{w}$ consists of all $F \in L_{2}(X)$ such that, if $f(g)=\int \bar{F} T(g) F d m-\left|\int F d m\right|^{2}$ for all $g \in G$, then $M(|f|)=0$. See Bergelson and Rosenblatt [3] for a proof of the above using the work of Godement, or see Krengel $[14$, p. 111], where this theorem is discussed in relationship to the work of Jacobs, Deleeuw and Glicksberg.

The representation $T$ is weakly mixing if and only if $L_{2}(X)_{c}$ consists of just the constants. Of particular importance in $\S 2$ are some of the structural aspects of $L_{2}(X)_{c}$. In case $G$ is abelian, in particular $G=Z$ as in Halmos [11], the space $L_{2}(X)_{c}$ has an orthonormal basis $\left(F_{\lambda}: \lambda \in \Lambda\right)$ of eigenvectors. That is $\left(F_{\lambda}: \lambda \in \Lambda\right)$ is an orthonormal basis of $L_{2}(X)_{c}$ such that for all $g \in G$, there exists $c_{\lambda}(g) \in C$ such that $T(g) F_{\lambda}=c_{\lambda}(g) F_{\lambda}$ a.e. [m]. Also, $T$ is ergodic if and only if the constant functions are the only eigenvectors with eigenvalue one. If $T$ is ergodic and $\lambda_{1}, \lambda_{2} \in \Lambda$ are such that $c_{\lambda_{1}}(g)=c_{\lambda_{2}}(g)$ for all $g \in G$, then $F_{\lambda_{3}}=F_{\lambda_{2}}$ a.e. $[m]$. That is, the 
eigenvalue homomorphisms $c_{\lambda}: G \rightarrow T$ are simple. The analogues of these results for general groups are described in Propositions 1.1 and 1.4.

1.1. Proposition. The representation $T$ restricted to $L_{2}(X)_{c}$ decomposes as an orthogonal direct sum of finite-dimensional subrepresentations.

There are a number of proofs of this theorem. See [3] for a discussion of the proof via Godement's decomposition of positive definite functions. Also, see Dye [6] for a proof for amenable groups that can be generalized to any locally compact group. These proofs all suggest that there should be a direct argument using only the Peter-Weyl theorem for compact groups. Indeed, in Deleeuw and Glicksberg [4, p. 72], the necessary fact about compactifications is observed which is needed in the proof of the following:

1.2. TheOREM. Suppose $G$ is a group of unitary transformations $\left\{V_{\mathrm{g}}: g \in G\right\}$ of a Hilbert space $H$. Then a necessary and sufficient condition for $H$ to decompose as a direct sum of $G$-invariant finite-dimensional subspaces is that for all $F \in H,\left\{V_{\mathrm{g}} F: g \in\right.$ $G\}$ is totally bounded in the $L_{2}$-norm topology.

Proof. Because orbits $\left\{V_{g} F: g \in G\right\}$ are totally bounded when $F$ lies in a $G$-invariant finite-dimensional subspace of $H$, the condition is necessary. Conversely, suppose all orbits are precompact. Let $\mathscr{G}$ be the weak-operator closure of $G$ in the bounded operators $B(H)$. Give $\mathscr{G}$ the weak-operator topology.

Theorem 3.2 in Deleeuw and Glicksberg [3] proves that $\mathscr{G}$ is a compact semigroup with a jointly continuous multiplication. Clearly, if $A \in \mathscr{G}$, then $A^{*} \in \mathscr{G}$, too. But also $A^{*}=A^{-1}$. That is, $\mathscr{G}$ consists of unitary transformations. Indeed, suppose $A=\lim _{i} V_{g_{i}}$ in the weak-operator topology. Then $\lim _{i} V_{g_{i}-1}=A^{*}$ in that topology and so

$$
\begin{aligned}
I & =\lim _{i} V_{g_{i}} V_{g_{i}-1}=\left(\lim _{i} V_{g_{i}}\right)\left(\lim _{i} V_{g_{i}-1}\right) \\
& =\left(\lim _{i} V_{g_{i}-1}\right)\left(\lim _{i} V_{g_{i}}\right) \\
& =A A^{*}=A^{*} A
\end{aligned}
$$

by the joint continuity of the multiplication. Finally, this also shows that $A \rightarrow A^{-1}=$ $A^{*}$ is continuous in $\mathscr{G}$. Thus, $\mathscr{G}$ is a compact group, continuing $\left\{V_{g}: g \in G\right\}$ as a dense subgroup, and acts continuously as unitary transformations on $H$.

Now we apply the Peter-Weyl principle (as in Greenleaf and Moskowitz [11] or in Hewitt and Ross [13, p. 29]) to argue that $H$ is a direct sum of $\mathscr{G}$-invariant, and hence $G$-invariant, finite-dimensional subspaces.

Remark 1. Notice that the positive definite functions arising from the representation $T$ on $L_{2}(X)_{c}$ (or of $G$ on $H$ in 1.2) are almost-periodic. Hence, the almost periodic compactification of a group as in Loomis [15] can be used to envelop the representation and prove 1.2 analogously to the above. Also, the uniqueness part of theorem 27.44 [13] applies to the decomposition and hence each finite-dimensional $T$ invariant $T$-irreducible subspace $A \subset L_{2}(X)_{c}$ has a multiplicity $\mu=\mu(H, T)$ associated with it. As in [13, p. 29], for $\sigma \in \hat{\mathscr{G}}$, let $M_{\sigma}$ be the smallest closed subspace of $L_{2}(X)_{c}$ containing all such $A$ with $\mathscr{G}$ equivalent to $\sigma$ on $A$. This subspace will be used in the proof of 1.6 . 
Remark 2. Let $\mathscr{G}_{c}$ be the enveloping compact group as in the proof of 1.2 for $\{T(g): g \in G\}$ when $H=L_{2}(X)_{c}$. Let $\beta_{c}$ be $\left\{A \in \beta: 1_{A} \in L_{2}(X)_{c}\right\}$. It is easy to show $\beta_{c}$ is a $\sigma$-algebra too and the largest compact factor in $\beta$, see [3] and [10]. Also, $L_{2}(X)_{c}$ is naturally isometric to $L_{2}\left(X, \beta_{c}, m\right)$ since each $F \in L_{2}(X)_{c}$ is $\beta_{c}$-measurable. For any $A \in \mathscr{G}_{c}$, there exists $\left(g_{i}\right) \subset G$ such that for all $F_{1}, F_{2} \in L_{2}(X)_{c}$, $\lim _{i}\left\langle T\left(g_{i}\right) F_{1}, F_{2}\right\rangle=\left\langle A\left(F_{1}\right), F_{2}\right\rangle$ and $\lim _{i}\left\langle T\left(g_{i}^{-1}\right) F_{1}, F_{2}\right\rangle=\left\langle A^{-1}\left(F_{1}\right), F_{2}\right\rangle$. Hence, $\left(T\left(g_{i}\right): i\right)$ is Cauchy in the weak topology of $\mathcal{M}\left(X, \beta_{c}\right)$, cf. Halmos [12]. Thus, $\mathscr{G}_{c}$ as a group is identical to $\left\{T(g): g \in G_{c}\right\}$ where $G_{c}$ is the closure of $T(G)$ in $\mathcal{M}\left(X, \beta_{c}\right)$ in the weak topology and $T$ denotes the regular representation on $L_{2}(X)_{c}$. Moreover, since $\mathscr{G}_{c}$ is compact, this identification gives a topological isomorphism of $\mathscr{G}_{c}$ with the weak-operator topology into $G_{c}$ with the weak topology of $\mu\left(X, \beta_{c}\right)$. If the probability space $(X, \beta, m)$ is not standard, this may only identify $\mathscr{G}_{c}$ with Boolean $\sigma$-isomorphisms in $\mathcal{M}\left(X, \beta_{c}\right)$. To have $\mathscr{G}_{c}$ realized completely as point transformations as in Mackey [17] requires some further separability hypotheses on $G$ and/or $(X, \beta, m)$.

1.3. Definition. Assume $G$ has a countable dense subset. A separable (measurepreserving) representation of $G$ in $\mathcal{M}(X)$ is a representation $T$ of $G$ in $M(X)$ such that $(X, \beta, m)$ is a non-atomic separable probability space.

1.4. Proposition. Suppose $T$ is an ergodic separable representation of $G$ in $M(X)$ and let $H$ be a finite-dimensional T-invariant T-irreducible subspace of $L_{2}(X)_{c}$. Then the multiplicity $\mu_{H}$ of $H$ in $L_{2}(X)_{c}$ is at most $\operatorname{dim}(H)$.

Proof. The hypothesis on $G$ guarantees that there is a countable subgroup $G_{0}$ which is dense in $G$ and so $\left\{T(g): g \in G_{0}\right\}$ is a countable dense subset of $\mathscr{G}_{c}$. The decomposition of $L_{2}(X)_{c}$ into finite-dimensional $T$-invariant $T$-irreducible subspaces is completely determined by $\mathscr{G}_{c}$, and hence, because $T$ is continuous, by the representation $T$ restricted to $G_{0}$. Because $G_{0}$ is countable, we may assume without affecting this decomposition, up to unitary equivalence, that $(X, \beta, m)$ is a standard probability space. Hence, corollary 2 of Zimmer [20] applies and shows that there is a standard probability space $(Y, \Gamma, p)$ and an action of $G_{0}$ on it as measure preserving transformation so that the action of $G_{0}$ on $L_{2}(X)_{c}$ is equivalent to the action on $L_{2}(Y, \Gamma, p)$. Now Mackey [16], theorem 1, applies to the $G_{0}$-space $(Y, \Gamma, p)$. This shows that all $\mu_{H}$ are finite. Actually, an examination of the proof in [16] shows that the action of $\mathscr{G}_{c}$ on $L_{2}(X)_{c}$ is equivalent to the regular action of $\mathscr{G}_{c}$ on $L_{2}\left(\mathscr{G}_{c} / K\right)$ for a suitable closed subgroup $K$ of $\mathscr{G}_{c}$. Hence, the Peter-Weyl Theorem for $\mathscr{G}_{c}$ shows that any finite-dimensional $T$-invariant $T$-irreducible subspace $H$ of $L_{2}(X)_{c}$ has multiplicity no larger than $\operatorname{dim}(H)$.

1.5. Corollary. Suppose $T$ is an ergodic separable representation of $G$ in $M(X)$. Then there is $F \in L_{2}(X)_{c}$ such that span $\{T(g) F: g \in G\}$ is norm dense in $L_{2}(X)_{c}$.

Proof. Proposition 1.4 shows that the hypotheses of theorem 1.10 in Greenleaf and Moskowitz [11] are satisfied. This theorem gives exactly the above.

Questions. (1) When does there also exist a cyclic vector for $L_{2}(X)_{w}$ or $L_{2}(X)$ for ergodic separable group actions? (2) If $T$ is an ergodic separable action, when does 
there exist $A \in \beta$ such that the smallest $\sigma$-algebra containing $\{T(g) A: g \in G\}$ is $\beta$ up to null sets?

Another general property of the finite-dimensional decomposition of $L_{2}(X)_{c}$ is the following.

1.6. Proposition. Let $T$ be an ergodic separable representation of $G$ in $M(X)$. Then there is an orthogonal direct-sum decomposition of $L_{2}(X)_{c}$ into finite-dimensional T-invariant subspaces $H_{i}$ such that each $H_{i} \subset L_{\infty}(X)$ and hence each $H_{i}$ has an orthonormal basis formed by $L_{\infty}$-functions.

Proof. As in proof of theorem 1.2, there is a compact group $\mathscr{G}$ of unitary transformations of $L_{2}(X)_{c}$ such that $\{T(g): g \in G\}$ is dense in $\mathscr{G}$ in the weak-operator topology on $L_{2}(X)_{c}$. The proof of theorem 27.44 [13] first constructs a projection $P_{\sigma}: L_{2}(X)_{c} \rightarrow$ $M_{\sigma}$ for each $\sigma \in \hat{\mathscr{G}}$. The definition of $P_{\sigma}$ shows that for all $F_{1}, F_{2} \in L_{\infty}(X) \cap L_{2}(X)_{c}$,

$$
\left|\int P_{\sigma}\left(F_{1}\right) F_{2} d m\right| \leq d_{\sigma}^{2}\left\|F_{1}\right\|_{\infty}\left\|F_{2}\right\|_{1}
$$

since

$$
\sup \left\{\left|X_{\bar{\sigma}}(g)\right|: g \in \mathscr{G}\right\} \leq d_{\sigma} .
$$

Since $L_{\infty}(X) \cap L_{2}(X)_{c}$, is dense in $L_{1}\left(X, \beta_{c}, m\right)$, this shows $\left\|P_{\sigma}\left(F_{1}\right)\right\|_{\infty} \leq d_{\sigma}^{2}\left\|F_{1}\right\|_{\infty}$. Also, $P_{\sigma}$ maps $L_{\infty}(X) \cap L_{2}(X)_{c}$ onto a dense subspace of $M_{\sigma}$. Thus, $L_{\infty}(X) \cap M_{\sigma}$ is dense in $M_{\sigma}$ for all $\sigma \in \hat{\mathscr{G}}$. Since $M_{\sigma}$ is finite-dimensional by proposition 1.4 , this is enough to prove this proposition.

Remark. It may well be that this theorem is true without the assumption that $T$ is a separable action of $G$ in $M(X)$. Indeed, the extreme case is where $G$ is a compact group and $T$ is the action by left multiplication on $\left(G, \beta_{\lambda}, \lambda\right)$ where $\lambda$ is a leftinvariant Haar measure on $G$. There $L_{2}(G)_{c}=L_{2}(G)$ and this does have a basis of $L_{\infty}(G)$ functions as above because the coefficient functions of finite-dimensional irreducible representations of $G$ can be used in this role.

Yet another application of proposition 1.4 is this generalization of the well-known fact that if $T, S \in \mathcal{M}(X)$ and $T S=S T$, then $T$ ergodic and $S$ weakly mixing implies $T$ is weakly mixing too.

1.7. Proposition. Suppose $S$ is a weakly mixing representation of $G$ and $T$ is an ergodic separable representation of $G$ in $M(X)$. If $S$ and $T$ commute, then $T$ is weakly mixing too.

Proof. If $T$ were not weakly mixing, then there would exist a finite-dimensional $T$-invariant subspace $H \subset L_{2}^{0}(G)_{c}$, the mean zero functions in $L_{2}(X)_{c}$. We may assume $H$ is $T$-irreducible, For $g \in G, S(g) H$ is $T$ irreducible and the representation of $T$ on $S(g) H$ is equivalent to the representation $T$ on $H$. By the property of $M_{\sigma}$ where $\sigma$ is $T$ restricted to $H, S(g) H \subset M_{\sigma}$. Thus, $M_{\sigma}$ is an $S$ invariant subspace which is finite-dimensional by proposition 1.4 . Hence, $S$ cannot be weakly mixing.

There are other theorems that can be generalized along these lines. A particularly important one in relation to criteria used in $\$ 2$ is to determine when a tensor product 
$T \otimes S$ is ergodic. As in [3], if $S$ is weakly mixing and $T$ is ergodic, then $T \otimes S$ is ergodic. More generally, in the abelian case, $T \otimes S$ is ergodic if $T$ and $S$ are ergodic and share no common eigenvalues other than 1 . The following generalization of this fact holds.

1.8. Proposition. If $T$ and $S$ are ergodic representations, then $T \otimes S$ is ergodic if and only if $T$ and $S$ have disjoint spectra: there are no finite-dimensional subspaces $H_{1}$, $H_{2} \subset L_{2}^{0}(X)$ with $H_{1} T$-invariant and $H_{2} S$-invariant, such that $\left.T\right|_{H_{1}}$ is equivalent to $\left.S\right|_{H_{2}}$.

Proof. Suppose $\left.T\right|_{H_{1}}$ is equivalent to $\left.S\right|_{H_{2}}$. Then the contragradient representation of $\left.T\right|_{H_{1}}$ is realized as the complex conjugate $\left.S\right|_{\bar{H}_{2}}$. Since the trivial representation is a subrepresentation of $\left.\left.T\right|_{H_{1}} \otimes S\right|_{\bar{H}_{2}}$, the trivial representation is a subrepresentation of $T \otimes S$.

On the other hand, suppose $T$ and $S$ do have disjoint spectra. Let $M$ denote the unique $G$-invariant mean on WAP $(G)$. Suppose $0 \neq \mathscr{F} \in L_{2}^{0}(X \times X)$ is $T \otimes$ $S$-invariant. Then

$$
\left\langle M(g), \int_{X \times X}(T(g) \otimes S(g) \mathscr{F}) \bar{F} d m \times d m\right\rangle=\|\mathscr{F}\|_{2}^{2} \neq 0 .
$$

But for all $\varepsilon>0$, there exist $c_{i} \in C$ and $F_{i}, G_{i} \in L_{2}(X), i=1, \ldots, n$ such that for each $i, \int F_{i} d m$ or $\int G_{i} d m=0$, and $\left\|\mathscr{F}-\sum_{i=1}^{n} c_{i} F_{i} \otimes G_{i}\right\|_{2}<\varepsilon$. If we show

$$
\left\langle M(g), \int_{\mathrm{X} \times \mathbf{X}}\left(T(g) \otimes S(g) F_{i} \otimes G_{i}\right) \overline{F_{i} \otimes G_{i}} d m \times d m\right\rangle=0
$$

for all $i, j=1, \ldots, n$, letting $\varepsilon \rightarrow 0$ this would show $\|\mathscr{F}\|_{2}^{2}=0$, a contradiction which would prove $T \otimes S$ is ergodic.

Suppose then $F_{1}, F_{2}, G_{1}, G_{2} \in L_{2}(X)$ and for $i=1,2, \int F_{i} d m$ or $\int G_{i} d m$ is 0 . Let $L_{2}^{T}(X)_{c} \oplus L_{2}^{T}(X)_{w}$ and $L_{2}^{S}(X)_{c} \oplus L_{2}^{S}(X)_{w}$ be the orthogonal decompositions for $S$ and $T$ discussed earlier, and write $F_{i}=F_{i}^{c}+F_{i}^{w}, G_{i}=G_{i}^{c}+G_{i}^{w}$ for $i=1,2$ according to this decomposition. Since $T$ is weakly mixing on $L_{2}^{T}(X)_{w}$ and $S$ is weakly mixing on $L_{2}^{S}(X)_{w}$, we have

$$
\begin{aligned}
&\left\langle M(g), \int_{X \times X}\left(T(g) \otimes S(g) F_{1} \otimes G_{1}\right) \overline{F_{2} \otimes G_{2}} d m \times d m\right\rangle \\
&=\left\langle M(g), \int_{X \times X}\left(T(g) \otimes S(g) F_{1}^{c} \otimes G_{2}^{c}\right) \overline{F_{2}^{c} \otimes G_{2}^{c}} d m \times d m\right\rangle .
\end{aligned}
$$

See [3], $\S 1$, for further discussion. Hence, we may assume at the outset that $F_{i} \in L_{2}^{T}(X)_{c}$ and $G_{i} \in L_{2}^{S}(X)_{c}$ for $i=1,2$.

But now what we want to show is that $T_{c} \otimes S_{c}$ is ergodic where $T_{c}$ is $T$ restricted to the mean zero functions in $L_{2}^{\top}(X)_{c}$ and $S_{c}$ is $S$ restricted to the mean zero functions in $L_{2}^{S}(X)_{c}$. For representations of a locally compact group that are direct sums of finite-dimensional representations, the trivial representation is a subrepresentation of $T_{c} \otimes S_{c}$ if and only if for some subrepresentation of $T_{c}^{\prime}$ of $T_{c}$, the contragradient representation $\check{T}_{c}^{\prime}$ is a subrepresentation of $S_{c}$ (up to unitary equivalence). Because the representations here are obtained from non-singular group actions on a measure space, $\check{T}_{c}^{\prime}$ is just given by the complex conjugate $\bar{T}_{c}^{\prime}$ of $T_{c}^{\prime}$. 
So if some $S_{c}^{\prime}$ is equivalent to $\check{T}_{c}^{\prime}$, then $\bar{S}_{c}^{\prime}$ is equivalent to $T_{c}^{\prime}$. But $\bar{S}_{c}^{\prime}$ is a subrepresentation of $S_{c}$, and so the disjointness of the spectra of $S$ and $T$ prevents this. Hence $T_{c} \otimes S_{c}$ is ergodic and so $T \otimes S$ is ergodic.

In the above, if $\left.T\right|_{H_{1}}$ is equivalent to $\left.S\right|_{H_{2}}$ with $H_{1}$ having an orthonormal basis $F_{1}, \ldots, F_{n} \in L_{2}^{0}(X)$, and $V: H_{1} \rightarrow H_{2}$ is a unitary transformation such that $V T(g)=$ $S(g) V$ for all $g \in G$, then

$$
\phi\left(\xi_{1}, \xi_{2}\right)=\sum_{i=1}^{n} F_{i}\left(\xi_{i}\right) \overline{V F_{i}}\left(\xi_{2}\right)
$$

for $\xi_{1}, \xi_{2} \in X$ is a non-zero mean zero $T \otimes S$-invariant function. This explicit proof of the first part of the above is observed in Moore [17]; it shows how the trivial representation is a subrepresentation of $\left.\left.T\right|_{H_{1}} \otimes S\right|_{H_{2}}$.

The same argument as above can be used to prove a local version of this theorem when $T \otimes S$ is not ergodic. That is, let $F_{1} \in L_{2}^{T}(X)_{c}$ and $F_{2} \in L_{2}^{S}(X)_{c}$, both mean zero. Then

$$
\left\langle M(g), \int_{X} T(g) F_{1} \bar{F}_{1} d m \int S(g) F_{2} \overline{F_{2}} d m\right\rangle=0
$$

if and only if the positive definite functions $f_{i}(g)=\int_{X} T(g) F_{i} \bar{F}_{i} d m$ give rise to representations of $G$ with disjoint spectra.

\section{Averaging theorems}

The idea used in proving the major convergence theorem here is to use an abstract version of the van der Corput inequality, cf. [1]. To do this for amenable groups requires an approximate tiling lemma for averages in $G$ over Følner sequences. Let $|\cdot|$ be a fixed right invariant Haar measure on $G$. For simplicity, let $d \lambda_{G}(g)$ be denoted $d g$.

In this section integrals of the form $\int_{K_{m}} T(g) F d g$ are needed. The explanation of the meaning of $\int_{K_{m}} T(g) F d g$ when $G$ is not discrete and $K_{m}$ is compact is that it represents the usual Pettis integral of $g \rightarrow T(g) F \in L_{2}(X)$. Here $p: g \rightarrow T(g) F$ is continuous since it is weakly continuous and so its range is separable by the $\sigma$-compactness of $G$. So the weak continuity of $p$ shows that $p$ is $\lambda_{G}$-measurable by the Pettis measurability theorem, see Dunford and Schwartz [5, III.6.11]. Thus, this integral can also be taken to be the Bochner integral. The following propositions are well-known.

2.1. Proposition. Let $G$ be an amenable group and let $\left(K_{m}\right)$ be a right Folner sequence of compact sets. Let $A: G \rightarrow H$ be a function where $H$ is a Hilbert space. Assume $A(G)$ is bounded. Let

$$
S_{1}(m)=\left(1 /\left|K_{m}\right|\right) \int_{K_{m}} A(g) d g
$$

and

$$
S_{2}(m, h)=\left(1 /\left|K_{m}\right|\right) \int_{K_{m}}\left(1 /\left|K_{h}\right|\right) \int_{K_{h}} A(g z) d z d g
$$


Then, for all $h$,

$$
\lim _{m \rightarrow \infty}\left\|S_{1}(m)-S_{2}(m, h)\right\|=0 .
$$

In the sequel $\left(K_{m}\right)$ will be a Følner sequence chosen as above.

2.2. Proposition. If $T: G \rightarrow \mathcal{M}(X)$ is an ergodic representation of $G$, and

$$
\begin{gathered}
A_{m}(F)=\left(1 /\left|K_{m}\right|\right) \int_{K_{m}} T(g) F d g, \quad \text { then for all } F \in L_{p}(X), 1 \leq p<\infty, \\
\qquad \lim _{m \rightarrow \infty}\left\|A_{m} F-\int F d m\right\|_{p}=0 .
\end{gathered}
$$

Now suppose $T_{1}, \ldots, T_{n}$ are commuting representations of $G$ in $\mathcal{M}(X)$ and consider products $S_{j}(g)=T_{1}(g) \ldots T_{j}(g), j=1, \ldots, n$. For $F_{1}, \ldots, F_{n} \in L_{\infty}(X)$, the product $S_{1}(g) F_{1} S_{2}(g) F_{2} \ldots S_{n}(g) F_{n}$ is a well-defined element of $L_{\infty}(X)$. It will be important that $S_{j}$ is a representation of $G$, too, so it is necessary to assume that the $T_{j}$ are commuting in the sense that $T_{i}(g) T_{j}(h)=T_{j}(h) T_{i}(g)$ for $i \neq j$ and $g, h \in G$. It might seem that the theorems to follow could be phrased and proved for products $S_{1} F_{1} \ldots S_{n} F_{n}$ without knowing each $S_{i}$ is itself a product. However, we will need to use the fact that $S_{j}^{-1} S_{k}, j<k$, is a representation of $G$; and generally, if $S_{1}, S_{2}$ are representations of $G$ such that $S_{1}^{-1} S_{2}$ is a representation of $G$, then $S_{2}=S_{1} T$ for some representation $T$ of $G$ commuting with $S_{1}$. For these reasons, we generally assume $S_{i}$ are formed as above from commuting representations $T_{i}$. Either notation is appropriate in the case of abelian groups $G$.

2.3. Definition. We say $\left(T_{1}, \ldots, T_{n}\right)$ is mutually ergodic if $T_{1} \otimes T_{1} T_{2} \otimes \cdots \otimes T_{1} \ldots T_{n}$ is ergodic. We say $\left(T_{1}, \ldots, T_{n}\right)$ is fully mutually ergodic if $\left(T_{j}, \ldots, T_{n}\right)$ is mutually ergodic for all $j=1, \ldots, n$. This type of joint ergodicity of $T_{1}, \ldots, T_{n}$ should be contrasted with the one in [1].

2.4. THEOREM. Let $T_{1}, \ldots, T_{n}$ be commuting fully mutually ergodic representations of an amenable group $G$ in $\mathcal{M}(X)$. Let $S_{i}=T_{i} \cdots T_{i}$ for $i=1, \ldots, n$. Let $\left(K_{m}\right)$ be a right Folner sequence in $G$ and let $F_{1}, \ldots, F_{n} \in L_{\infty}(X)$. Then

$$
\lim _{m \rightarrow \infty}\left\|1 /\left|K_{m}\right| \int_{K_{m}} S_{1}(g) F_{1} \cdots S_{n}(g) F_{n} d g-\int F_{1} d m \cdots \int F_{n} d m\right\|_{L_{2}(X)}=0 .
$$

Proof. The proof is by induction on $n$. If $n=1$, we are assuming $T_{1}$ is ergodic, and proposition 2.2 proves the theorem. Assume the theorem has been proved for a fully mutually ergodic system $\left(T_{1}, \ldots, T_{n-1}\right)$. Assume $T_{1}, \ldots, T_{n}$ is fully mutually ergodic. Without loss of generality, $\int F_{1} d m=0$. Indeed, suppose the theorem is proved in case $\int F_{1} d m=0$. Then let $E\left(F_{1}\right)=\int F_{1} d m$. We have

$$
\begin{aligned}
S_{1}(g) & \left(F_{1}-E\left(F_{1}\right)\right) S_{2}(g) F_{2} \cdots S_{n}(g) F_{n} \\
& =S_{1}(g) F_{1} S_{2}(g) F_{2} \cdots S_{n}(g) F_{n}-E\left(F_{1}\right)\left(S_{2}(g) F_{2} \cdots S_{n}(g) F_{n}\right) .
\end{aligned}
$$

Since $\left(T_{1}, \ldots, T_{n}\right)$ is mutually ergodic, $\left(T_{1} T_{2}, T_{3}, \ldots, T_{n}\right)$ is mutually ergodic. By induction,

$$
\left(1 /\left|K_{m}\right|\right) \int_{K_{m}} S_{2}(g) F_{n} \cdots S_{n}(g) F_{n} d g \rightarrow \prod_{i=2}^{m} \int F_{i} d m
$$


as $m \rightarrow \infty$ in $L_{2}(X)$-norm. Thus,

$$
\lim _{m \rightarrow \infty}\left(1 /\left|K_{m}\right|\right) \int_{K_{m}} E\left(F_{1}\right) S_{2}(g) F_{2} \cdots S_{n}(g) F_{n} d g=\prod_{i=1}^{m} \int F_{i} d m
$$

in $L_{2}(X)$-norm, too. Hence, (6) shows the theorem follows from the case $E\left(F_{1}\right)=0$.

We assume $\int F_{1} d m=0$ and show

$$
\lim _{m \rightarrow \infty}\left(1 /\left|K_{m}\right|\right) \int_{K_{m}} S_{1}(g) F_{1} S_{2}(g) F_{2} \cdots S_{n}(g) F_{n} d g=0
$$

in $L_{2}(X)$-norm. If we let

$$
\begin{gathered}
A(g)=S_{1}(g) F_{1} S_{2}(g) F_{2} \cdots S_{n}(g) F_{n}, \\
H=L_{2}(X)
\end{gathered}
$$

in proposition 2.1, we see that it suffices to show that for all $\varepsilon>0$, there is an $h \geq 1$ such that for some $M \geq 1$ if $m \geq M$, then

Now

$$
\mathcal{N}=\left\|\left(1 /\left|K_{m}\right|\right) \int_{K_{m}}\left(1 /\left|K_{h}\right|\right) \int_{K_{h}} S_{1}(g z) F_{1} \cdots S_{n}(g z) F_{n} d z d g\right\|_{2}^{2}<\varepsilon
$$

$$
\mathcal{N} \leq\left(1 /\left|K_{m}\right|\right) \int_{K_{m}}\left\|\left(1 /\left|K_{h}\right|\right) \int_{K_{h}} S_{1}(g z) F_{1} \cdots S_{n}(g z) F_{n} d z\right\|_{2}^{2} d g .
$$

Indeed, if $A: G \rightarrow L_{2}(X)$ is bounded and weakly measurable, then for $K \subset G, K$ compact

$$
\begin{aligned}
\left\|\int_{K} A(g) d g\right\|_{2}^{2} & \leq\left(\int_{K}\|A(g)\|_{2} d g\right)^{2} \\
& \leqq\left[\left(\int_{K}\|A(g)\|_{2}^{2} d g\right)^{1 / 2}\left(\int_{K} 1 d g\right)^{1 / 2}\right]^{2}=|K| \int_{K}\|A(g)\|_{2}^{2} d g
\end{aligned}
$$

But we have,

$$
\begin{aligned}
&\left\|\left(1 /\left|K_{h}\right|\right) \int_{K_{h}} S_{1}(g z) F_{1} \cdots S_{n}(g z) F_{n} d z\right\|_{2}^{2} \\
&=\left(1 /\left|K_{h}\right|^{2}\right) \int_{K_{h}} \int_{K_{h}}\left(\int_{X} S_{1}(g s) F_{1} \cdots S_{n}(g s) F_{n} S_{1}(g t) \bar{F}_{1} \cdots S_{n}(g t) \bar{F}_{n} d m\right) d s d t \\
&=\left(1 /\left|K_{h}\right|^{2}\right) \int_{K_{h}} \int_{K_{h}}\left(\int_{X} T_{1}(s) F_{1}\left(T_{1}(s) T_{2}(g s) F_{2}\right) \cdots\left(T_{1}(s) T_{2}(g s) \cdots T_{n}(g s) F_{n}\right)\right. \\
&\left.\quad \times T_{1}(t) \bar{F}_{1}\left(T_{1}(t) T_{2}(g t) \bar{F}_{2}\right) \cdots\left(T_{1}(t) T_{2}(g t) \cdots T_{n}(g t) \bar{F}_{n}\right) d m\right) d s d t \\
&=\left(1 /\left|K_{h}\right|^{2}\right) \int_{K_{h}} \int_{K_{h}}\left(\int_{X}\left(T_{1}(s) F_{1}\right) T_{2}(g)\left[T_{1}(s) T_{2}(s) F_{2}\right] \cdots T_{2}(g) T_{3}(g) \cdots\right. \\
& \quad \times\left(T_{n}(g)\left[T_{1}(s) T_{2}(s) \cdots T_{n}(s) F_{n}\right]\left(T_{1}(t) \bar{F}_{1}\right) T_{2}(g)\left[T_{1}(t) T_{2}(t) \bar{F}_{2}\right] \cdots\right. \\
&\left.\times T_{2}(g) T_{3}(g) \cdots T_{n}(g)\left[T_{1}(t) \cdots T_{n}(t) \bar{F}_{n}\right] d m\right) d s d t \\
&=\left(1 /\left|K_{h}\right|^{2}\right) \int_{K_{h}} \int_{K_{h}}\left(\int_{X}\left[S_{1}(s) F_{1} S_{1}(t) \bar{F}_{1}\right]\left(T_{2}(g)\left[S_{2}(s) F_{2} S_{2}(t) \bar{F}_{2}\right]\right) \cdots\right. \\
& \times\left(T_{2}(g) \cdots T_{n}(g)\left[S_{n}(s) F_{n} S_{n}(t) \bar{F}_{n}\right] d m\right) d s d t .
\end{aligned}
$$


Hence, the induction hypothesis applied to the fully mutually ergodic system $\left(T_{2}, \ldots, T_{n}\right)$ shows that for all $h \geqslant 1$,

$$
\begin{aligned}
\lim _{m \rightarrow \infty}( & \left.1 /\left|K_{m}\right|\right) \int_{K_{m}} \|\left(1 /\left|K_{h}\right| \int_{K_{h}} S_{1}(g z) F_{1} \cdots S_{n}(g z) F_{n} d z \|_{2}^{2} d g\right. \\
= & \left(1 /\left|K_{h}\right|^{2}\right) \int_{K_{h}} \int_{K_{h}} \int_{X} S_{1}(s) F_{1} S_{1}(t) \bar{F}_{1} d m \int_{X} S_{2}(s) F_{2} . \\
& \left.\times S_{2}(t) \bar{F}_{2} d m \cdots \int_{X} S_{n}(s) F_{n} S_{n}(t) \bar{F}_{n} d m\right) d s d t \\
= & \left\|1 /\left|K_{h}\right| \int_{K_{h}} S_{1}(g) \otimes \cdots \otimes S_{n}(g)\left(F_{1} \otimes \cdots \otimes F_{n}\right)\right\|_{L_{2}\left(X^{n}\right)}^{2}
\end{aligned}
$$

But $S_{1} \otimes \cdots \otimes S_{n}$ is ergodic and so for any $\varepsilon>0$, there is an $h>1$ such that this last norm is no larger than $\varepsilon$ because

$$
\int_{\mathrm{X}} \cdots \int_{\mathrm{X}} F_{1} \otimes \cdots \otimes F_{n} d m \cdots d m=\prod_{l=1}^{n} \int_{X} F_{l} d m=0 .
$$

For this $h$, there is an $M \geq 1$ such that for all $m \geq M, \mathcal{N} \leq 2 \varepsilon$.

2.5. Examples. (a) Suppose $G$ and $X$ are separable as in definition 1.3. Then assume $T_{1}, \ldots, T_{n}$ are commuting representations of $G$ such that $T_{1}$ is weakly mixing and each $T_{i}$ is ergodic. By proposition 1.7, each $T_{i}$ is weakly mixing. Thus, if also each $S_{i}$ is ergodic (and hence weakly mixing by the same argument), then $S_{1} \otimes \cdots \otimes S_{n}$ is weakly mixing. It follows that $\left(T_{1}, \ldots, T_{n}\right)$ is fully mutually ergodic. Indeed, without the separability assumption, if each $T_{i}$ and $S_{i}$ is weakly mixing, then $\left(T_{1}, \ldots, T_{n}\right)$ is fully mutually ergodic. This situation is the generalization of a weakly mixing system of commuting transformations as in [9]. But as in [1] where the exact hypotheses for (3) were given, the hypotheses for theorem 2.4 above are less than assuming that all $T_{i}$ and $S_{i}$ are weakly mixing. In fact, theorem 2.6 will show that the hypotheses of theorem 2.4 are essentially necessary, thus generalizing the work in [1].

(b) Let $H=\bigoplus_{i=1}^{n} G$, with the product topology. Any representation $T: H \rightarrow L$ on a Hilbert space $L$ gives commuting representations $T_{i}: G \rightarrow L$ defined by $T_{i}(g)=$ $T\left(e_{i}(g)\right)$ where $e_{i}: G \rightarrow H$ is the $i$ th-coordinate injection. If $T$ is strongly mixing, then so is each $T_{i}$. The proof of theorem 2.5 in Bergelson and Rosenblatt [3] shows, for amenable groups $G$ among others, that in the weak topology, the representations $T$ such that each $T_{i}$ and $S_{i}$ is weakly mixing from a residual subset of all the representations of $H$ on $L$. So, at the unitary level, there are many representations $T$ of $H$ such that $\left(T_{1}, \ldots, T_{n}\right)$ is fully mutually ergodic as representations of $G$ as unitary operators on $L$. Using the Gaussian measure space construction, see Neveu [18] or Schmidt [19], each such $T$ and system $\left(T_{1}, \ldots, T_{n}\right)$ gives a representation $T: H \rightarrow M(X)$ such that $\left(T_{1}, \ldots, T_{n}\right)$ is fully mutually ergodic as point transformations. Hence, for any group $G$, there are examples of systems $\left(T_{1}, \ldots, T_{n}\right)$ where the hypotheses theorem 2.4 hold. If $G$ is discrete, it is easier to give an example. Let $X=\prod_{H}[0,1]$ and let $H$ act on $X$ by permutation of the coordinate indices. 
Then in the product probability measure given by Lebesgue measure in $[0,1]$, each $T_{i}$ and $S_{i}$ is strongly mixing.

(c) If $G$ is abelian, then the existence of eigenvalues and orthonormal bases of eigenvectors for a representation $T$ in $\mathcal{M}(X)$ restricted to $L_{2}(X)_{c}$ allows a simplification of the hypotheses of theorem 2.4. It is not too hard to show that if $T_{1}, \ldots, T_{n}$ are ergodic and $S_{1} \otimes S_{2} \otimes \cdots \otimes S_{n}$ is ergodic, then $T_{2} \otimes T_{2} T_{3} \otimes \cdots \otimes$ $T_{2} \ldots T_{n}$ is ergodic. So, if $T_{1}, \ldots, T_{n}$ and $S_{1} \otimes S_{2} \otimes \cdots \otimes S_{n}$ are ergodic, then $\left(T_{1}, \ldots, T_{n}\right)$ is fully mutually ergodic. Thus, by proposition 1.8 , when $G$ is abelian, $\left(T_{1}, \ldots, T_{n}\right)$ is fully mutually ergodic if and only if just the constants have the eigenvalue 1 for $T_{i}$ and if $\Lambda_{i}$ denotes the set of eigenvalues of $S_{i}$, then for $\lambda_{i} \in \Lambda_{i}$, $1=1, \ldots, n, \lambda_{1} \lambda_{2} \ldots \lambda_{n}=1$ if and only if $\lambda_{i}=1$ for all $i$. Of course, this includes the case where $\Lambda_{i}=\varnothing$ for all $i$, i.e. each $T_{i}$ is weakly mixing. Is there a simplification of the hypotheses of theorem 2.5 along these lines for general groups?

One of the interesting aspects of the hypotheses of theorem 2.4 is that they are necessary given $T_{1}, \ldots, T_{n}$ commute.

2.6. THEOREM. Let $T_{1}, \ldots, T_{n}$ be commuting, separable representations of an amenable group $G$ in $M(X)$ and fix a right Folner sequence $\left(K_{m}\right)$. Suppose that for all $F_{0}, \ldots, F_{n} \in L_{\infty}(X)$;

$$
\lim _{m \rightarrow \infty} \int_{X}\left(1 /\left|K_{m}\right| \int_{K_{m}} F_{0} S_{1}(g) F_{1} \ldots S_{n}(g) F_{n} d g\right) d m=\prod_{i=0}^{n} \int_{X} F_{i} d m .
$$

Then $S_{1} \otimes S_{2} \otimes \cdots \otimes S_{n}$ is ergodic. Also, $\left(T_{1}, \ldots, T_{n}\right)$ is fully mutually ergodic.

Proof. Only the ergodicity of $S_{1} \otimes \cdots \otimes S_{n}$ is needed to get the rest. Indeed, let $F_{1}=1$. Then

$$
\begin{aligned}
\int_{X}\left(1 /\left|K_{m}\right| \int_{K_{m}} S_{1}(g) F_{1} \cdots S_{n}(g) F_{n} d g\right) d m \\
=\left(1 /\left|K_{m}\right|\right) \int_{K_{m}}\left(\int_{X} S_{2}(g) F_{2} \cdots S_{n}(g) F_{n} d m\right) d x \\
=\left(1 /\left|K_{m}\right|\right) \int_{K_{m}}\left(\int_{X}\left(T_{2}(g) F_{2}\right) \cdots\left(T_{2}(g) \ldots T_{n}(g) F_{n} d m\right) d g\right. \\
=\int_{X}\left(1 /\left|K_{m}\right| \int_{K_{m}} \mathscr{S}_{1}(g) F_{2} \ldots \mathscr{S}_{n-1}(g) F_{n} d g\right) d m,
\end{aligned}
$$

where $\mathscr{Y}_{i}=T_{2}, \ldots, T_{i+1}, i=1, \ldots, n-1$. Hence, (7) holds for $\left(\mathscr{S}_{1}, \ldots, \mathscr{S}_{n-1}\right)$. So, by induction, we would get $\left(T_{1}, \ldots, T_{n}\right)$ being fully mutually ergodic. Note also that (7) entails the ergodicity of each $T_{i}$ and $S_{i}$. For example, fix $i=1, \ldots, n$ and let $F_{j}=1$ for $j \in\{i-1, i\}$. Then (7) implies

$$
\lim _{m \rightarrow \infty}\left(1 /\left|K_{m}\right|\right) \int_{X}\left(\int_{K_{m}} F_{i-1} T_{i}(g) F_{i}(g) d m\right)=\int F_{i-1} d m \int F_{i} d m .
$$

Hence, if $F \in L_{\infty}(X)$ is $T_{i}$-invariant, then

$$
\int_{X}\left|F_{i}\right|^{2} d m=\left|\int_{X} F_{i} d m\right|^{2}
$$


by taking $\overline{F_{i-1}}=F_{i}=F$. So, if $A \in \beta$ is $T_{i}$-invariant, then $m(A)=m(A)^{2}$ and $m(A)$ is 0 or 1 .

Let $L_{2}(X)=L_{c}^{i} \oplus L_{w}^{i}$ where $L_{c}^{i}$ is the compact summand for $S_{i}$ and $L_{w}^{i}$ is the weakly mixing summand for $S_{i}$. We identify $L_{2}\left(X^{n}\right)$ with $\otimes_{i=1}^{n} L_{2}(X)$ in the usual fashion. On an invariant subspace of the form $L_{2}(X) \otimes \cdots \otimes L_{w}^{i} \otimes \cdots \otimes L_{2}(X)$, $S_{1} \otimes \cdots \otimes S_{n}$ is ergodic because $S_{i}$ is weakly mixing on $L_{i}^{w}$. So, if $S_{1} \otimes \cdots \otimes S_{n}$ has an invariant function, it must be in $L_{c}^{1} \otimes \cdots \otimes L_{c}^{n}$. But the existence of an $S_{i}$-invariant finite-dimensional orthogonal direct sum decomposition of $L_{c}^{i}$ with an $L_{\infty}$ basis on the summands is guaranteed by proposition 1.6. Hence, if $S_{1} \otimes \cdots \otimes S_{n}$ is not ergodic, then there exist $S_{i}$-invariant finite-dimensional subspaces $H_{i}$ of $L_{c}^{i} \cap L_{\infty}(X)$, with some $H_{i} \subset L_{2}^{0}(X)$, such that some $0 \neq F \in H_{1} \otimes \cdots \otimes H_{n} \subset L_{2}^{0}(X)$ is invariant under $S_{1} \otimes \cdots \otimes S_{n}$. Let $\left(f_{k}^{i}: k=1, \ldots, \operatorname{dim}\left(H_{i}\right)\right)$ be orthonormal bases in $L_{\infty}(X)$ for each $H_{i}$. Then $F$ has unique expansion as a finite sum

$$
F\left(\xi_{1}, \ldots, \xi_{n}\right)=\sum_{\left(m_{1}, \ldots, m_{n}\right)} a\left(m_{1}, \ldots, m_{n}\right) f_{m_{1}}^{1}\left(\xi_{1}\right) \cdots f_{m_{n}}^{n}\left(\xi_{n}\right)
$$

for a.e. $\left(\xi_{1}, \ldots, \xi_{n}\right) \in X^{n}$.

For each $g \in G,\left.S_{i}(g)\right|_{H_{i}}$ has a matrix expansion $S_{i}(g)=\left[\alpha_{m n}^{i}\right]$ in the basis $\left(f_{m}^{i}: m\right)$ with $\alpha_{m n}^{i}$ depending on $g$. Since $\left(S_{1} \otimes \cdots \otimes S_{n}\right) F=F$, for all $g \in G$,

$$
\begin{aligned}
F & =\sum_{\left(k_{1}, \ldots, k_{n}\right)} a\left(k_{1}, \ldots, k_{n}\right)\left(\sum_{l_{1}} \alpha_{l_{1} k_{1}}^{1} f_{l_{1}}^{1}\right) \cdots\left(\sum_{l_{n}} \alpha_{l_{n} k_{n}}^{n} f_{l_{n}}^{n}\right) \\
& =\sum_{\left(k_{1}, \ldots, k_{n}\right)} \sum_{\left(l_{1}, \ldots, l_{n}\right)} a\left(k_{1}, \ldots, k_{n}\right) \alpha_{l_{1} k_{1}}^{1} \cdots a_{l_{n} k_{n}}^{n} f_{l_{1}}^{1} \cdots f_{l_{n}}^{n} .
\end{aligned}
$$

Hence, for all $\left(l_{1}, \ldots, l_{n}\right)$,

$$
a\left(l_{1}, \ldots, l_{n}\right)=\sum_{\left(k_{1}, \ldots, k_{n}\right)} a\left(k_{1}, \ldots, k_{n}\right) \alpha_{l_{1} k_{1}}^{1} \cdots \alpha_{l_{n} k_{n}}^{n} .
$$

But then for a.e. $\xi \in X$, and all $g \in G$,

$$
\begin{aligned}
& \sum_{\left.k_{1}, \ldots, k_{n}\right)} a\left(k_{1}, \ldots, k_{n}\right) S_{1}(g) f_{k_{1}}^{1}(\xi) \ldots S_{n}(g) f_{k_{n}}^{n}(\xi) \\
& \quad=\sum_{\left(k_{1}, \ldots, k_{n}\right)} a\left(k_{1}, \ldots, k_{n}\right)\left(\sum_{l_{1}} a_{l_{1} k_{1}}^{1} f_{l_{1}}^{1}(\xi)\right) \cdots\left(\sum_{l_{n}} \alpha_{l_{n} k_{n}}^{n} f_{l_{n}}^{n}(\xi)\right) \\
& \quad=\sum_{\left(k_{1}, \ldots, k_{n}\right)} \sum_{\left(l_{1}, \ldots, l_{n}\right)}^{\sum} a\left(k_{1}, \ldots, k_{n}\right) \alpha_{l_{1} k_{1}}^{1} \cdots \alpha_{l_{n} k_{n}}^{n} f_{l_{1}}^{1}(\xi) \ldots f_{l_{n}}^{n}(\xi) \\
& \quad=\sum_{\left(l_{1}, \ldots, l_{n}\right)} a\left(l_{1}, \ldots, l_{n}\right) f_{l_{n}}^{1}(\xi) \cdots f_{l_{n}}^{n}(\xi) .
\end{aligned}
$$

Fix $F_{0} \in L_{2}(X)$. We have

$$
\begin{aligned}
& \int_{X} F_{0}(\xi)\left(\sum_{\left(l_{1}, \ldots, l_{n}\right)} a\left(l_{1}, \ldots, l_{n}\right) f_{l_{1}}^{1}(\xi) \cdots f_{l_{n}}^{n}(\xi)\right) d m(\xi) \\
& =\left(1 /\left|K_{m}\right|\right) \int_{K_{m}} \int F_{0}(\xi) \cdot \sum_{\left(l_{1}, \ldots, l_{n}\right)} a\left(l_{1}, \ldots, l_{n}\right) S_{1}(g) f_{l_{1}}^{1}(\xi) \cdots S_{n}(g) f_{l_{n}}^{n}(\xi) d m(\xi) d g \\
& =\sum_{\left(l_{1}, \ldots, l_{n}\right)}^{\sum} a\left(l_{1}, \ldots, l_{n}\right)\left(\int\left(1 /\left|K_{m}\right|\right) \int_{K_{m}} F_{0}(\xi) S_{1}(g) f_{l_{1}}^{1}(\xi) \cdots S_{n}(g) f_{l_{n}}^{n}(\xi) d g d m(\xi)\right.
\end{aligned}
$$


By (7), letting $m \rightarrow \infty$, this shows for all $F_{0} \in L_{\infty}(X)$,

$$
\int F_{0}\left(\sum_{\left(l_{1}, \ldots, l_{n}\right)} a\left(l_{1}, \ldots, l_{n}\right) f_{l_{1}}^{1} \cdots f_{i_{n}}^{n}\right) d m=0
$$

because for some $i$, all $f_{m}^{i}$ are mean zero. That is,

a.e. $\xi[m]$.

$$
\sum_{\left(l_{1}, \ldots, l_{n}\right)} a\left(l_{1}, \ldots, l_{n}\right) f_{l_{1}}^{1}(\xi) \cdots f_{l_{n}}^{n}(\xi)=0
$$

Now, if

$$
g_{1}, \ldots, g_{n-1} \in G, T_{2}\left(g_{1}\right) \otimes T_{3}\left(g_{2}\right) \otimes \cdots \otimes T_{n}\left(g_{n-1}\right) \otimes I
$$

commutes with $S_{1} \otimes \cdots \otimes S_{n}$. So, $\left(T_{2}\left(g_{1}\right) \otimes \cdots \otimes T_{n}\left(g_{n-1}\right) \otimes I\right) F$ is $S_{1} \otimes \cdots \otimes S_{n}$ invariant, too. Moreover, $T_{i+1}\left(g_{i}\right) H_{i}, i=1, \ldots, n-1$, is $S_{i}$-invariant with orthonormal basis $\left(T_{i+1}\left(g_{i}\right) f_{m}^{i}: m\right)$. So, the same argument as the one above shows that for all $g_{1}, \ldots, g_{n-1} \in G$,

$$
0=\sum_{\left(l_{1}, \ldots, l_{n}\right)} a\left(l_{1}, \ldots, l_{n}\right) T_{2}\left(g_{1}\right) f_{l_{1}}^{1}(\xi) \cdots T_{n}\left(g_{n-1}\right) f_{l_{n-1}}^{n-1}(\xi) f_{l_{n}}^{n}(\xi)
$$

a.e. $\xi[m]$. But each $T_{i}$ is ergodic, and so

$$
\lim _{m \rightarrow \infty} 1 /\left|K_{m}\right| \int_{K_{m}} T_{i}(g) F d x=\int F d m
$$

in $L_{2}(X)$-norm. Hence, using (8),

$$
\begin{aligned}
0= & \lim _{m \rightarrow \infty}\left(1 /\left|K_{m}\right|^{n-1}\right) \int_{K_{m}} \cdots \int_{K_{m}} \\
& \times \int_{\left(l_{1}, \ldots, l_{n}\right)} a\left(l_{1}, \ldots, l_{n}\right) T_{2}\left(g_{1}\right) f_{l_{1}}^{1}(\xi) \cdots T_{n}\left(g_{n-1}\right) f_{l_{n-1}}^{n-1}(\xi) f_{l_{n}}^{n}(\xi) \\
& \times \sum_{\left(k_{1}, \ldots, k_{n}\right)} a\left(\overline{l_{1}, \ldots, l_{n}}\right) T_{2}\left(g_{1}\right) \overline{f_{l_{1}}^{1}(\xi)} \cdots T_{n}\left(g_{n-1}\right) \overline{f_{l_{n-1}}^{n-1}(\xi) \overline{f_{l_{n}}^{n}}(\xi) d m(\xi) d g_{1} \cdots d g_{n-1}} \\
= & \sum_{\left(l_{1}, \ldots, l_{n}\right)} \sum_{\left(k_{1}, \ldots, k_{n}\right)} a\left(l_{1}, \ldots, l_{n}\right) \overline{a\left(k_{1}, \ldots, k_{n}\right)} E\left(f_{l_{1}}^{1} \overline{f_{k_{1}}^{1}}\right) \cdots E\left(f_{l_{n}}^{n} \overline{f_{k_{n}}^{n}}\right) .
\end{aligned}
$$

By the orthonormality of $\left(f_{m}^{j}\right)$, this gives

$$
0=\sum_{\left(l_{1}, \ldots, l_{n}\right)}\left|a\left(l_{1}, \ldots, l_{n}\right)\right|^{2} .
$$

So all $a\left(l_{1}, \ldots, l_{n}\right)=0$ and $F=0$, a contradiction.

Remark. Theorems 2.4 and 2.6 show that the seemingly weaker joint ergodicity of (7) is equivalent to (5) for commuting separable actions $T_{1}, \ldots, T_{n}$. Also, the proof could have been shorter if we knew that the bases $\left(f_{m}^{j}: m\right)$ could have been chosen to be generic, i.e. not only are $\left(f_{m_{1}}^{1}\left(\xi_{1}\right) \cdots f_{m_{n}}^{n}\left(\xi_{n}\right):\left(m_{1}, \ldots, m_{n}\right)\right)$ orthonormal in $L_{2}\left(X^{n}\right)$, but $\left(f_{m_{1}}^{1}(\xi) \cdots f_{m_{n}}^{n}(\xi):\left(m_{1}, \ldots, m_{n}\right)\right)$ are linearly independent. If $G$ is abelian, this could be arranged, but it is not clear if it is always possible. See [8] for other uses of genericity.

There is another possible definition of joint ergodicity that one might use as a generalization of (3). For instance, we might assume for all $F_{0}, \ldots, F_{n} \in L_{\infty}(X)$ that

$$
\lim _{m \rightarrow \infty}\left(1 /\left|K_{m}\right|\right) \int_{K_{m}}\left|\int_{X} F_{0} S_{1}(g) F_{1} \ldots S_{n}(g) F_{n}-\prod_{i=0}^{n} \int_{X} F_{i} d m\right|^{2} d g=0 .
$$


This property is related to (5) and (7) as in [10, p. 534]. However, taking all $F_{i}=1$, $i \geq 1$, except for $F_{j}$, shows that property (9) forces $S_{j}$ to be weakly mixing and so $S_{1} \otimes \cdots \otimes S_{n}$ is ergodic, too. Thus, if one wishes to get a joint ergodicity result that does not entail all representations being weakly mixing, then (5) or (7) are better forms to study.

Acknowledgment. We would like to thank Professor Furstenberg very much for his help in completing the proof of theorem 2.6. We also thank the referee for many helpful suggestions and comments.

\section{REFERENCES}

[1] D. Berend \& V. Bergelson. Jointly ergodic measure preserving transformations. Israel J. Math. 49 (1984), 307-314.

[2] D. Berend \& V. Bergelson. Characterization of joint ergodicity for non-commuting transformations. Israel J. Math. 56 (1986), 123-8.

[3] V. Bergelson \& J. Rosenblatt. Mixing actions of groups, to appear in Illinois $J$. Math.

[4] K. Deleeuw \& I. Glicksberg. Applications of almost periodic compactifications. Acta Math. 105 (1961), 63-97.

[5] N. Dunford \& J. Schwartz. Linear Operators Vol. I. Interscience, New York, 1964.

[6] H. Dye. On the ergodic mixing theorem. TAMS 118 (1965), 123-130.

[7] H. Furstenberg. Ergodic behavior of diagonal measures and a theorem of Szemeredi on arithmetic progressions. J. d'Analyse Math. 31 (1977), 204-256.

[8] H. Furstenberg. Recurrence in Ergodic Theory and Combinatorial Number Theory Princeton University Press, Princeton, New Jersey, 1981.

[9] H. Furstenberg \& Y. Katznelson. An ergodic Szemeredi theorem for commuting transformations. J. d'Analyse 34 (1978), 275-291.

[10] H. Furstenberg, Y. Katznelson \& D. Ornstein. The ergodic theoretical proof of Szemeredi's theorem. BAMS 7 (1982), 527-552.

[11] F. Greenleaf \& M. Moskowitz. Cyclic vectors for representations of locally compact groups. Math. Annalen 190 (1971), 265-288.

[12] P. Halmos. Lectures in Ergodic Theory. Math. Soc. of Japan, Kenkyusha, Tokyo, 1956.

[13] E. Hewitt \& K. Ross. Abstract Harmonic Analysis Vol. II, Springer-Verlag, New York, Heidelberg, Berlin, 1970.

[14] V. Krengel. Ergodic Theorems Walter de Cruyter, Berlin, New York, 1985.

[15] L. Loomis. An Introduction to Abstract Harmonic Analysis Van Nostrand, Toronto, New York, London, 1953.

[16] G. Mackey. Ergodic transformation groups with a pure point spectrum. Ill. J. Math.8 (1964), 593-600.

[17] C. C. Moore. Ergodicity of flows on homogeneous spaces. Amer. J. Math. 88 (1960), 154-178.

[18] J. Neveu. Processes Aléatoires Gaussiens Presses V. of Montreal, Montreal, 1968.

[19] K. Schmidt. Asymptotic properties of unitary representations and mixing. Proc. London Math. 48 (1984), 445-460.

[20] R. Zimmer. Extensions of ergodic group actions. Ill. J. Math. 20 (1976), 373-409. 